\title{
AN ASSESSMENT OF IMAGING MODELS FOR EXIT WAVE RESTORATION
}

\author{
A I Kirkland, L. Y. Chang \\ Oxford University, Department of Materials, Parks Road, Oxford, OX1 3PH, UK
}

Indirect restoration (for a review see [1]) of the specimen exit plane wavefunction from either focal $[2,3]$ or tilted illumination [4] series of images is now widespread due to the availability of field emission sources and accurately characterised CCD detectors [5]. These techniques have the twin advantages of improving the interpretable resolution through indirect compensation of the lens aberrations and providing complex data for structural interpretation.

The general expression for the Fourier transform of the intensity in an electron microscope image is given by the sum over the pairs of diffracted beams:

$$
I(\mathbf{k})=\sum_{\mathbf{k}^{\prime}} \Psi\left(\mathbf{k}+\mathbf{k}^{\prime}\right) \Psi^{*}\left(\mathbf{k}^{\prime}\right) T\left(\mathbf{k}+\mathbf{k}^{\prime}, \mathbf{k}^{\prime}\right)
$$

where $\Psi$ is the Fourier transform of the object transmission function, and $\mathrm{T}$ is the transmission cross coefficient which accounts for all lens aberrations, limiting coherence effects (both spatial, $\mathrm{E}_{\mathrm{s}}$ and temporal, $\mathrm{E}_{f}$ ) and the Modulation Transfer Function (MTF) due to the recording system. For a weak scattering approximation, only those terms involving interference with the primary beam are considered, and so equation (1) reduces to

$$
I(\mathbf{k})=\Psi(\mathbf{k}) T(\mathbf{k})+\Psi^{*}(-\mathbf{k}) T^{*}(-\mathbf{k})
$$

This weak scattering approximation therefore gives a simple linear relationship between the image contrast and the specimen exit plane wavefunction. Hence when the object is sufficiently thin and the microscope dependent parameters are precisely measured, the specimen exit plane wavefunction may be recovered by an appropriate linear image restoration filter. Linear filters, being analytical have the advantage of computational efficiency whereas the more general non-linear restoration filters require computationally intensive numerical iteration. However at the higher resolutions that are now routinely achievable, particularly with the advent of aberration correction [6], interference between the scattered beams is no longer negligible and filters that correctly restore the non-linear image intensity are essential in many applications.

In this paper the use of linear (Wiener) and non-linear (Maximum likelihood) restoration methods are compared for a range of materials including perfect crystal and grain boundaries. The performance of both the linear Wiener filter and the nonlinear MAL method with linear and nonlinear image intensity was investigated following the procedure outlined in FIG 1 in which simulated specimen exit plane wavefunctions calculated using the multislice algorithm were imaged for both non-linear image intensity given in equation (1) and the linear image intensity given in equation (3) for a range of defoci. These two sets of through focus images were then restored using both filter types to obtain two approximations to the exit plane waves for comparison (FIG 2).

\section{References}

[1] Kirkland, A. I. and Meyer, R. R. (2004) Microscopy and Microanalysis, 10, 401

[2] Coene, W. M. J. et al., (1996) Ultramicroscopy 64, 109

[3] Coene, W. M. J. et al., (1992) Phys. Rev. Letts. 69, 3743

[4] Kirkland, A. I. et al., (1995) Ultramicroscopy 57, 355

[5] Meyer, R. R. and Kirkland, A. I. (2000) Microsc. Res. Tech., 49, 269 
[6] Haider et al., (1998) Ultramicroscopy 75, 53

[7] This research was supported by EPSRC and the Leverhulme Trust.

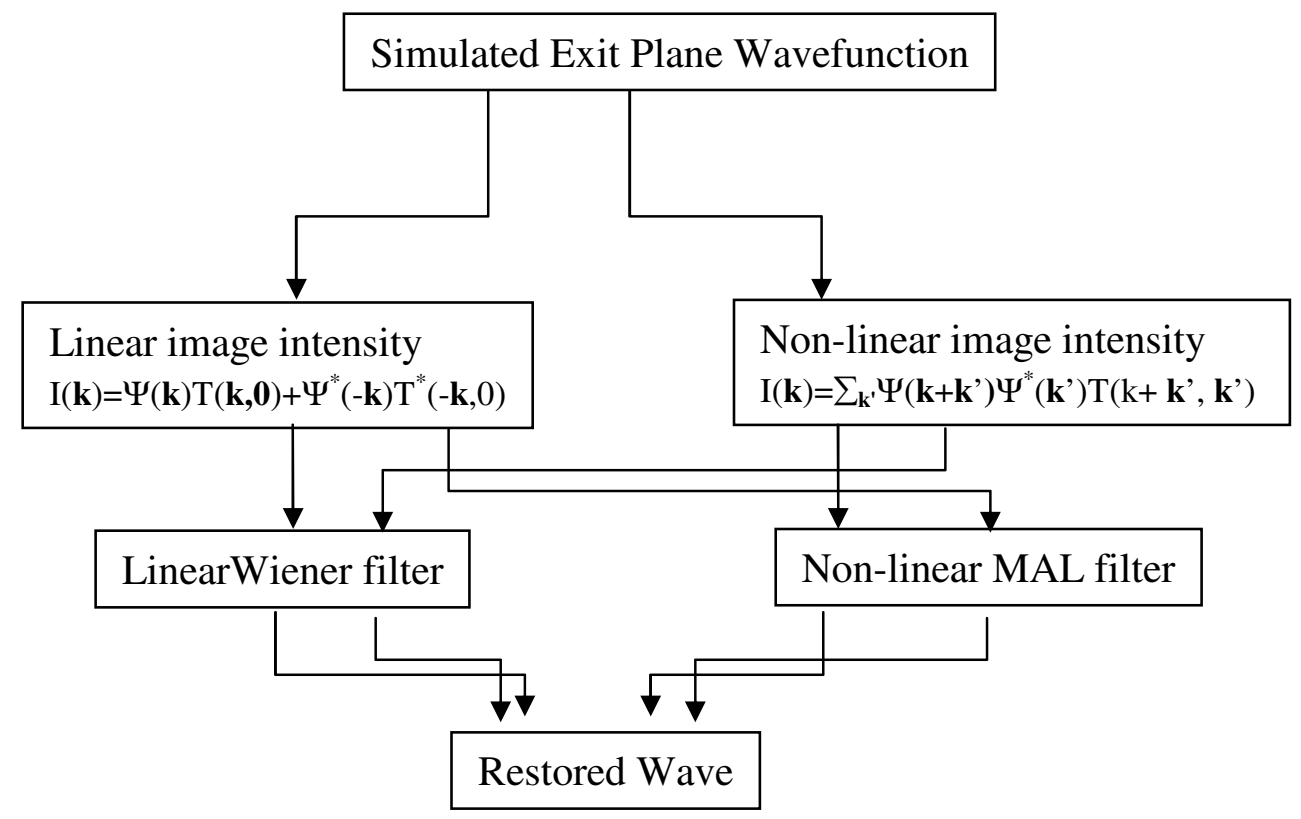

FIG. 1. Schematic diagram showing the procedure used for comparison of linear and non-linear restoration filters. .
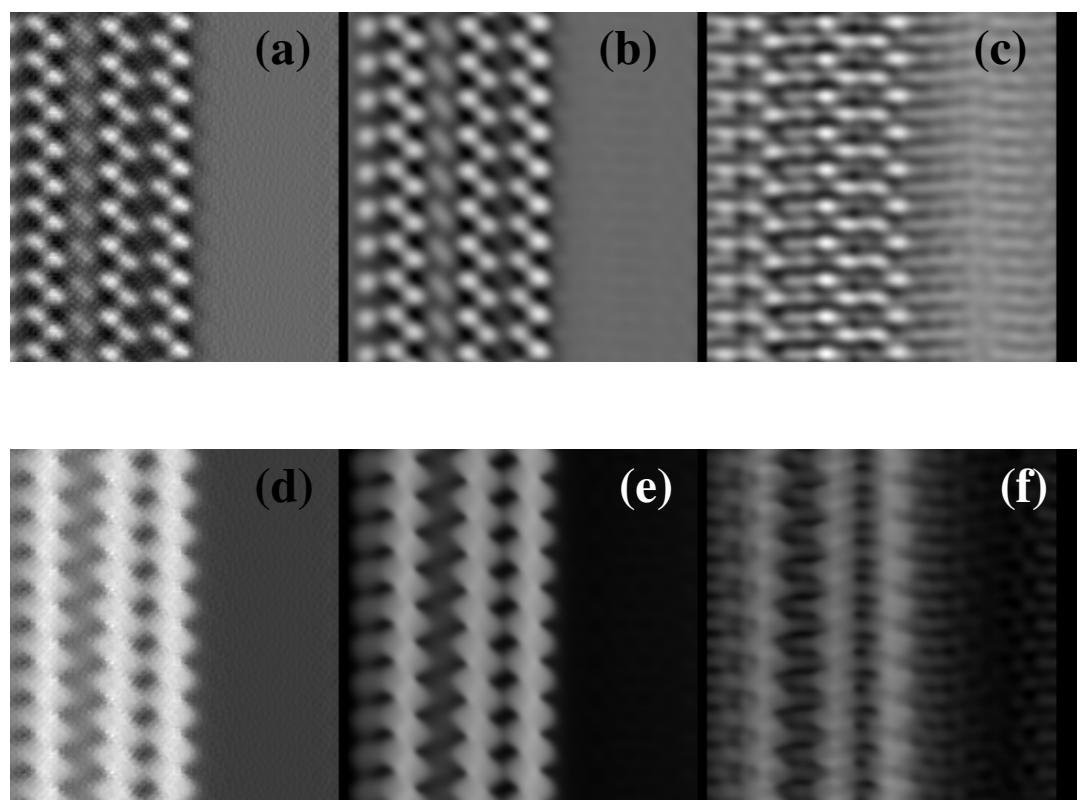

FIG 2 Modulus (a-c) and phase (d-f) of an input wavefunction of a crystal surface of $\mathrm{Si}_{3} \mathrm{~N}_{4}(\mathrm{a}$, d) and restored waves using a non-linear $(b, e)$ and linear $(c, f)$ filter. In the case of the wavefunction restored using the linear filter substantial delocalisation of the crystalline contrast occurs beyond the edge of the crystal. 